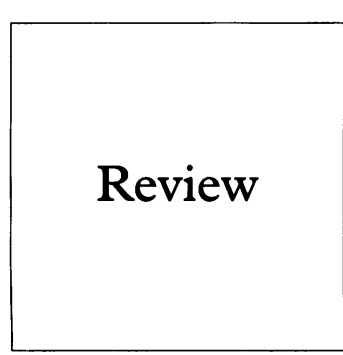

\title{
Epidemiological treatment and tests of cure in gonococcal infection: evidence for value
}

\author{
Chris A Carne
}

Objectives: To evaluate the evidence for value of using epidemiological treatment and of using $\underset{\mathcal{D}}{\mathbb{1}}$ tests of cure in the management of gonococcal infections. Methods: Medline was used to search the literature for well-conducted studies that had a bear- $\frac{\overrightarrow{\bar{N}}}{\mathcal{D}}$ ing on the evaluation of these issues.

Conclusions: Epidemiological treatment is treatment given to named contacts of patients after a history of exposure to disease but without or in advance of confirmatory pathological findings. $\frac{\mathscr{N}}{\mathbb{D}}$ It may be given when the clinician considers that the risk to the patient of unnecessary treatment $\stackrel{\Omega}{2}$ is outweighed by the risk of complications of the infection or the probability of transmission of the infection to other contacts. It may be appropriate where detection by microscopy is relatively $\vec{O}$ unreliable such as when infection of the cervix, rectum, or pharynx is suspected. If epidemiological treatment is given, then it does not obviate the need for confirmatory tests to be sent, tests of ${ }_{\sigma}^{\omega}$ cure to be done, or for contact tracing. At least one test of cure is mandatory following treatment $\stackrel{\mathscr{S}}{=}$ of gonorrhoea. The test of cure in women should include a blind rectal swab. For infection of $\omega$ the pharynx and rectum the chances of a false negative test of cure are higher and, therefore,.more than one test of cure is required. An additional test of cure is also necessary in patients $\vec{N}$ with salpingitis or disseminated gonococcal infection. This paper is a discussion of two interre- $\frac{\text { O }}{3}$ lated issues in the management of gonorrhoea. It is based on two presentations by the author at a workshop organised by Dr Mark Fitzgerald entitled Development of audit measures and guidelines $\mathbb{\Phi}$ for good practice in the management of gonorrhoea and held at the Royal College of Physicians, London, in May 1995. The conclusions reached are based on the consensus view of the partici- $\frac{\partial}{2}$ pants. For simplicity the two issues are dealt with consecutively. (Genitourin Med 1997;73:12-15)

Keywords: gonorrhoea; tests of cure; treatment of contacts

\section{Epidemiological treatment}

Introduction Various definitions of epidemiological treatment exist. The broader definition refers to "administration of antibiotics when the diagnosis is considered likely". ${ }^{1}$ For the purposes of this paper the following, narrower, definition is used, that is, "treatment given to named contacts of patients after a history of exposure to disease but without or in advance of confirmatory pathological findings". ${ }^{2}$ The rationale of using epidemiological treatment is "that the limitations of the available diagnostic procedures and the delays arising therefrom or due to attempting repeated tests, may nullify or reduce the value of the treatment ultimately given". ${ }^{3}$

In this paper three approaches are used to examine the evidence for the value of epidemiological treatment. Firstly, international comparisons; secondly, an evaluation of the major factors influencing the decision for and against epidemiological treatment; and lastly, a summary of two studies which have provided data for its direct evaluation. With one or two notable exceptions most of the studies which provide evaluable data to contribute to the debate were done at least ten years ago. The author does not believe that the implications of the studies would be different were they to be repeated today. Much useful information has been provided by the National Audit Development Project in Sexual Health. Nevertheless, further up-to-date well- conducted studies would provide a more secure foundation on which to base decisions $\frac{3}{3}$ on management.

International Comparisons It is unwise to give too much weight to international comparisons of when evaluating epidemiological treatment. Many other factors such as the nature of the health care system and the level of resources available are important in the control of sexually transmitted diseases. However, the experi-음 ence in Sweden suggests that the appropriate use of epidemiological treatment can contribute to lowering the incidence of gonor- $N$ rhoea.

Different countries have adopted the prac- N tice of epidemiological treatment with differ- 0 ing enthusiasm. Before 1970 Swedish venereologists and other physicians generally considered that because of legislation, treat- $?$ ment of gonorrhoea could not be started unless gonococci had been demonstrated by direct microscopy and/or by culture. From $\frac{\rho}{\Phi}$ 1970 onwards the Swedish public health $\frac{\varrho}{\circ}$ authorities also recommended treatment based on epidemiological information. According to Danielsson "the measures were certainly of importance to prevent further $\frac{0}{\partial}$ spread of gonococcal infections". ${ }^{4}$ The incidence of gonorrhoea in Sweden fell from 487 cases per 100000 in 1970 to 31 cases per 100000 in 1987.5

In the USA the Centers for Disease Control has recommended since 1950 that female con-

\footnotetext{
Addenbrooke's Hospital, Cambridge, CB2 2QQ, UK Accepted for publication 16 October 1996
} 
tacts of men with gonorrhoea receive epidemiological treatment, and in 1972 this policy was extended to include male contacts of culturepositive women. ${ }^{6}$ Gonorrhoea morbidity rates reached a peak in 1946 in the USA, decreased until 1957 and increased again for nearly two decades, peaking at 473 per 100000 in $1975 .^{5}$

A survey of gonorrhoea practices in Great Britain was conducted by the British Co-operative Clinical Group in 1968. This found that $69 \%$ of clinics treated females with epidemiological treatment at least in some cases, but only $19 \%$ of clinics did so for males. ${ }^{7}$ Data discussed later in this paper provide an explanation for this greater tendency to use epidemiological treatment for females than for males. The incidence of gonorrhoea in England has remained below 120 per 100000 for the past 30 years and more. It decreased to 30 per 100000 in $1995 .^{8}$

Factors for and against epidemiological treatment Prevention of morbidity in the patient. The higher the risk of morbidity arising in the contact the greater the argument for epidemiological treatment. The prevalence of gonorrhoea among female contacts of infected men ranges from $36 \%$ to $92 \%$ and in most studies usually runs between $45 \%$ and $60 \%$. The prevalence of gonorrhoea in the male sexual partners of infected women ranges from about $40 \%$ to $90 \% .{ }^{9}$ A nationwide survey in the UK in early 1995 found that the prevalence rates of gonorrhoea among female and male contacts were $60 \%$ and $74 \%$ respectively. (Fitzgerald $M$, Bedford $\mathrm{C}$ for the Central Audit Group in Genitourinary Medicine: The Clinical Management of Gonorrhoea. Results from the National Audit Development Project in Sexual Health. 1996). Pelvic inflammatory disease (PID) has been found to occur in $25 / 570$ $(4 \cdot 4 \%)^{10}$ to $61 / 606(10 \cdot 6 \%)^{11}$ of cases in UK studies. Delay in treatment appears to be important. It has been estimated (though no data are shown) that $15 \%$ of women with gonorrhoea will develop PID during the first month of their infection. ${ }^{9}$ One study found that of 124 female patients with gonorrhoea who were not treated at the initial visit and who required treatment at the second visit (at a mean interval of seven days, range 2 to 83 days), nine $(7 \cdot 3 \%)$ developed pelvic inflammatory disease during the interval between visits. ${ }^{12}$ Disseminated gonococcal infection is far less common and has been estimated to occur in about $1 \%$ of cases. ${ }^{13}$

Patient default rate A retrospective sample of female cases of gonorrhoea treated in clinics in England and Wales during 1978 found that "six per cent of contacts who had negative smear results at their initial visit, were not treated epidemiologically, but were subsequently proved to be infected by culture defaulted before treatment. Among patients who attended with a contact slip, however, the default rate was only $2 \%$ compared with $14 \%$ among those who gave a verbal history of exposure to gonorrhoea". ${ }^{14}$

Diagnostic difficulties: microscopy Epidemiological treatment is more likely to be used when there are difficulties in making the diag- nosis at the initial visit. This may apply particularly to developing countries. ${ }^{15}$ In the UK the ability to diagnose by microscopy varies according to the site being tested. Gonococcal urethritis in men is relatively easily diagnosed on microscopy, being found in $94.5 \%$ of cases. ${ }^{16}$ In contrast, women infected at the cervix or urethra or both, were only diagnosed on microscopy in $61.8 \%$ of cases in one study ${ }^{16}$ and $53.4 \%$ in another. ${ }^{10} \mathrm{~A}$ recent audit at one large London clinic showed that the sensitivity of cervical gram stained smears was only $29 \% .{ }^{17}$ Earlier work showed that rectal gonorrhoea in men was diagnosed by microscopy in $58 \%$ of cases. ${ }^{18}$ In 1995 the Central Audit Group in Genitourinary Medicine found that while $681 / 762(89 \%)$ of male urethral gonorrhoea was diagnosed on microscopy, only $152 / 407(37 \%)$ of gonorrhoea in females, and $20 / 49(41 \%)$ of rectal gonorrhoea in male homosexuals was diagnosed in this way. Rates of diagnosis by microscopy may, however, be higher when the patient is a known contact of gonorrhoea.

Diagnostic difficulties: culture If a single culture is a relatively insensitive means of diagnosing gonorrhoea at a specific site, clinicians may be more inclined to use epidemiological treatment. A combination of microscopy and culture performed on one occasion in a woman with gonorrhoea will miss the diagnosis in $3-9 \%$ of cases. ${ }^{1920}$ About $7 \%$ of men with rectal gonorrhoea may be missed by culture on only one occasion, and at least $25 \%$ with pharyngeal gonorrhoea will be missed on a single culture. ${ }^{18}$

Other issues which need to be considered concern the cost of the unnecessary treatment of uninfected contacts and the side effects caused. There may also be medico-legal problems arising from treating someone for a condition which they do not have. Furthermore, there may be a risk of lax standards developing where epidemiological treatment is used frequently.

Studies providing data for the direct evaluation of epidemiological treatment In a study of 604 cases of gonorrhoea in women 16 patients who were microscopy negative but culture positive with the first set of tests failed to return and were not treated. Only four of these 16 patients were contacts of men with gonorrhoea. The authors estimate that epidemiological treatment would have resulted in the unnecessary treatment of 142 patients, and in the treatment of the four infected contacts who defaulted but not the 12 patients in whom there was no reason to suspect gonorrhoea. ${ }^{10}$

In Belsey's study of practices in STD clinics in England and Wales in 1978, a sample of 2933 cases diagnosed, treated or returned as gonorrhoea was obtained. Exactly one-third of patients were not named contacts and thus could not have been given epidemiological treatment. Of the remaining patients who provided some evidence of a history of exposure to the disease, $24 \%$ were treated epidemiologically. Thirty-five per cent of cases treated epidemiologically were later proved to be true cases of gonorrhoea. ${ }^{14}$ It is worth bearing in 
mind, however, the insensitivity of a single culture. Belsey's figure of $35 \%$ being proved to be true cases may hide a further small number of true cases. Nevertheless, it appears that nearly two-thirds of patients treated epidemiologically did not, in fact, have gonorrhoea.

Conclusions Epidemiological treatment has advantages for the patient in cutting the risk of complications. On the other hand, it reduces the proportion of true cases of gonorrhoea in whom the diagnosis is confirmed because a single microscopy plus a single culture will miss some cases. If such cases are not used as index cases for contact tracing this will tend to mitigate against good control of gonorrhoea in the community. It is, therefore, advisable that contact tracing is conducted on all patients treated epidemiologically. If epidemiological treatment is to be employed the disadvantages are less when the contacts are truly untraceable. The case for epidemiological treatment is strengthened when microscopy and culture are insensitive or unreliable. There is, therefore, a stronger case for using epidemiological treatment when the patient is at risk of having infection at the pharynx, cervix or rectum because diagnostic techniques are less sensitive at these sites than at the urethra. Diagnostic techniques may, in general, be less reliable in parts of the developing world and, in the UK, when taken outside of genitourinary medicine clinics. Epidemiological treatment may, therefore, be more justifiable in these settings provided that it does not lead to the development of lax standards. In conclusion, epidemiological treatment may be given when the clinician considers that the risk to the patient of unnecessary treatment is outweighed by the risk of complications of the infection or the probability of transmission of the infection to other contacts. If epidemiological treatment is given, then it does not obviate the need for confirmatory tests to be sent, tests of cure to be done, or for contact tracing. ${ }^{21}$

\section{Tests of cure}

Introduction The rationale given for tests of cure is two-fold. Firstly, to prevent complications in the index case and, secondly, to minimise the risk of subsequent transmission of infection from the index case. Tests of cure may at times inadvertently pick up new infections which have been acquired since successful treatment of the initial episode. In such instances the same benefits accrue. This paper examines the evidence which may be used to assess the value of tests of cure under the following headings: biological factors; difficulties in diagnosis and treatment; and finally, studies attempting a direct evaluation.

Biological factors Prevention of complications and of transmission may be particularly important for strains of gonococci which have been shown to carry a risk of greater morbidity. The gonococci causing salpingitis are likely to have the following characteristics: non-Arg ${ }^{-} \mathrm{Hyx}^{-} \mathrm{Ura}^{-}$, higher-molecular weight protein I's and the WII serotype. ${ }^{22}$ Similarly, there are markers associated with gonococci which are prone to cause disseminated gono- coccal infection. ${ }^{22}$ There is a greater need for tests of cure for patients with these conditions.

Antibiotic-resistant strains of gonorrhoea pose a threat to the public health, especially if they show multiple resistance. For this reason there is an increased need for tests of cure for penicillinase producing Neisseria gonorrhoea (PPNG).

Difficulties in diagnosis and treatment If treat ment is known to carry a high risk of failure at a particular anatomic site, there is a greatero need for tests of cure. If diagnostic techniques are insensitive at making the initial diagnosis of gonococcal infection at a particular site those same techniques will again be insensitive when used as tests of cure.

Anatomic site of infection has been shown to be important when treating gonococcal infections. $^{23}$ In a systematic review of pub $\overrightarrow{0}$ lished therapeutic trials of various antimicrobial regimens for the biological cure of uncomplicated mucosal Neisseria gonorrhoea infections, the cure rates were calculated by anatomic site for 16,737 infections. The curérates were as follows: female urethra, $98.4 \% \overrightarrow{\mathrm{N}}$ male urethra, 96.4\%; cervix, $98 \%$; female pharynx, $83.7 \%$; male pharynx, $79 \cdot 2 \%$; female rectum, 97.9\%; and male rectum, $95 \cdot 3 \%$. The figures obtained by the Central Audit Group in the UK in early 1995 in a survey of $843^{2}$ gonococcal infections were as follows: female urethra, 97\%; male urethra, 96\%; cervix $97 \%$; female pharynx $8 / 9(89 \%)$; male pharynx, 91\%; female rectum, 95\%; male rectum $93 \%$. It may be argued, therefore, that the need for tests of cure is greatest for gonococcap infections of the pharynx.

Diagnosis of gonococcal infections may be insensitive in men infected at the pharynx of rectum. At least $25 \%$ of pharyngeal gonorrhoea in homosexual men will be missed on ap. single culture and about $7 \%$ of rectal gonor rhoea. ${ }^{19}$ Three to nine percent of cases of gon orrhoea in women may be missed by one set of tests (microscopy and culture). ${ }^{20} 21$

Studies to evaluate tests of cure A large study in the USA attempted to evaluate the need for post-treatment cultures in men with gonococcat urethritis. ${ }^{24}$ Because the large majority of men with gonorrhoea respond to antimicrobiab therapy it was hypothesised that a sympto- $N$ matic response would predict successful treat ment. The records of 4897 men witho gonorrhoea were studied. One hundred ando eighty three $(3.7 \%)$ were treatment failures on days 3-7 after treatment. The clinical response to therapy was correlated with microbiologica 5 outcome. Before therapy, 4662 men $(95 \cdot 2 \%)$ had dysuria and/or urethral discharge. Of the 582 men who remained symptomatic, $103^{\circ}$ $(17 \cdot 7 \%)$ had a positive culture after treatment as compared with only $78(1.9 \%)$ of 4080 meno who became asymptomatic $(p<0.01)$. The conclusion was that men who are initially? symptomatic and become asymptomatic after therapy have a small chance (one in 50) of being infected at the post-treatment visit. However, almost half ( 78 of $183,42.6 \%$ ) of treatment failures in men with gonococcal urethritis are asymptomatic. Therefore, a policy 
of confining post-treatment cultures to those who remain symptomatic would miss $42 \cdot 6 \%$ of treatment failures and this would have implications for the risk of developing complications and particularly for greater transmission of gonorrhoea. The authors concluded that "If resources allow, all patients with gonorrhoea should have a post-treatment culture". However, the cost implications are considerable. In 1976 Judson and Wolf calculated the cost of detecting a positive post-treatment culture. Using a calculated average cost per follow-up visit of $\$ 12 \cdot 75$, they worked out that it cost $\$ 427$ to detect a patient (male or female) with a positive culture. ${ }^{25} \mathrm{~A}$ later paper from the USA compared four alternative strategies for the management of men with acute urethritis. They calculated that test-ofcure cultures for patients who were asymptomatic after treatment for gonorrhoea required the expenditure of from $\$ 4900$ to $\$ 109800$ for each case of asymptomatic, persistent gonorrhoea discovered and cured. ${ }^{26}$

An American paper specifically evaluated rectal culture without the use of an "anoscope" as a test of cure of gonorrhoea in the female. ${ }^{27}$ The rectal and cervical sites were compared as a test of cure in 908 female patients who had gonorrhoea. After treatment, positive cervical or rectal cultures (or cultures of both sites) were found in $10.6 \%$ of the patients. Thirty per cent of the therapeutic failures would have been missed if only the cervical site had been tested. It was also found that antibiotic therapy is more likely to fail if Neisseria gonorrhoea infects both the cervix and the rectum.

Conclusions If tests of cure are not performed routinely on men treated for gonococcal urethritis, almost half $(42.6 \%)$ of treatment failures will be missed with important consequences for subsequent transmission. The case for tests of cure is even stronger for infection in men at other anatomic sites and infection in women. There is also a strong case for performing a blind rectal swab as a test of cure in women with gonorrhoea. For infection of the pharynx and rectum the chances of a false negative test of cure are higher and, therefore, more than one test of cure is required. An additional test of cure is also necessary in patients with salpingitis or disseminated gonococcal infection as they are likely to carry strains which are prone to cause these complications. The cost of performing tests of cure is high but this must be balanced against the costs generated by less effective control of gonorrhoea in the community. An up-to-date cost-benefit analysis would be useful.

1 Judson FN, Maltz AB. A rational basis for the epidemiologic treatment of gonorrhoea in a clinic for sexually transmitted diseases. Sex Transm Dis 1978;5:89-92.

2 Adler MW. Diagnostic, treatment and reporting criteria for gonorrhoea in sexually transmitted disease clinics in England and Wales: 2 Treatment and reporting criteria. Br ₹ Venereal Dis 1978;54:15-23.

3 Willcox R. "Epidemiological" treatment in venereal diseases other than syphilis. Brf Venereal Dis 1973;49:116-25.

4 Danielsson D. Gonorrhoea. In: Anglo-Scandinavian Congress on Sexually Transmitted Diseases. Oriel JD, Waugh MA, eds. London Royal Society of Medicine Waugh MA, eds.

5 Aral SO, Holmes KK. Epidemiology of sexual behaviour and sexually transmitted diseases. In: Sexually Transmitted Diseases. Holmes KK, Mardh P-A, Sparling PF, Wiesner PJ, Cates W, Lemon SM, Stamm WE, eds. New York: McGraw Hill. 2nd ed. 1990.

6 Blount $\mathrm{JH}$. A new approach for gonorrhoea epidemiology. Am ₹ Public Health 1978;62:710-2.

7 British Co-operative Clinical Group. Survey of gonorrhoea practices in Great Britain. Br F Venereal Dis 1971;47: practices

8 Statistical Bulletin. Sexually transmitted diseases, England 1995. Department of Health. Bulletin 1996/14

9 Rein MF. Epidemiology of gonococcal infections. In: The Gonococcus. Roberts RB, ed. London: John Wiley and Sons. 1977.

10 Barlow D, Phillips I. Gonorrhoea in women. Lancet 1978;1:761-4.

11 Rees E, Annels EH. Gonococcal salpingitis. $\mathrm{Br} f$ Venereal Dis 1978;45:205-15.

12 Lossick JG, Smeltzer MP, Curran JW. The value of the cervical gram stain in the diagnosis and treatment of gonorrhoea in women in a venereal disease clinic. Sex Transm Dis 1982;9:124-7.

13 Holmes KK, Wiesner PJ, Pedersen AHB. The gonococcal arthritis-dermatitis syndrome. Ann Intern Med 1971;75 470-1.

14 Belsey EM. Epidemiological treatment of gonorrhoea and non-specific genital infection in female sex contacts. $\mathrm{Br} \mathcal{F}$ Venereal Dis 1978;58:113-6.

15 Meheus AZ. Epidemiological treatment of gonorrhoea. Lancet 1973;2:678-9.

16 Goh BT, Varia KB, Ayliffe PF, Lim FKS. Diagnosis of gonorrhoea by gram-stained smears and cultures in men and women; role of the urethral smear. Sex Transm Dis 1985;12:135-9.

17 Evans JK, Mercey DE, French PD, Prince MV. Audit of a diagnosis of gonorrhoea at first visit to a London genitourinary medicine clinic. Genitourin Med 1994;70:291-2.

18 McMillan A, Young $H$. Gonorrhoea in the homosexual man: frequency of infection by culture site. Sex Transm Dis 1978;5:146-50

19 Barlow D, Nayyar K, Phillips I. Diagnosis of gonorrhoea in women. Br ₹ Venereal Dis 1976;52:326-8.

20 Chipperfield EJ, Catterall RD. Reappraisal of Gram-staining and cultural techniques for the diagnosis of gonorrhoea in women. $\mathrm{Br} \mathcal{F}$ Venereal Dis 1976;52:36-9.

21 Fitzgerald $M$, Bedford C. National standards for the management of gonorrhoea. Int $\mathcal{f}$ STD AIDS 1996;7: 298-300.

22 Brooks GF. Chapters on Salpingitis and Disseminated Gonococcal Infection. In: Gonococcal Infection. Brooks GF, Donegan EA, eds. London: Edward Arnold. 1985.

23 Moran JS. Treating uncomplicated Neisseria gonorrhoea infections: is the anatomic site of infection important? infections: is the anatomic site
Sex Transm Dis 1995;22:39-47.

24 Schmid GP, Johnson RE, Brenner ER. Symptomatic response to therapy of men with Gonococcal Urethritis do all need post-treatment cultures? Sex Transm Dis $1987 ; 14: 37-40$

25 Judson FN, Wolf FC. Re-screening for gonorrhoea: An evaluation of compliance methods and results. $A m \mathcal{F}$ Public Health 1979;69:1178-80.

26 Braun P, Sherman H, Komaroff AL. Urethritis in men: Benefits, risks, and costs of alternative strategies of manBenefits, risks, and costs of alternative strate

27 Schroeter AL, Reynolds G. The rectal culture as a test of cure of gonorrhoea in the female. $\mathcal{F}$ Infect Dis $1972 ; 125$ 499-503. 